\title{
Recovery of dicer-like 1-late flowering phenotype by miR172 expressed by the noncanonical DCL4-dependent biogenesis pathway
}

\author{
MASAYUKI TSUZUKI, ${ }^{1}$ ATSUSHI TAKEDA, ${ }^{2}$ and YUICHIRO WATANABE ${ }^{1}$ \\ ${ }^{1}$ Department of Life Sciences, Graduate School of Arts and Sciences, The University of Tokyo, Komaba 3-8-1, Meguro-ku, Tokyo 153-8902, Japan \\ ${ }^{2}$ Graduate School of Life Sciences, Ritsumeikan University, Noji-higashi 1-1-1, Kusatsu, Shiga 525-8577, Japan
}

\begin{abstract}
MicroRNAs (miRNAs) act as down-regulators of gene expression, and play a dominant role in eukaryote development. In Arabidopsis thaliana, DICER-LIKE 1 (DCL1) is the main processor in miRNA biogenesis, and dcl1 mutants show various developmental defects at the early stage of embryogenesis or at gamete formation. However, miRNAs responsible for the respective developmental stages of the $d c / 1$ defects have not been identified. Here, we developed a DCL1-independent miRNA expression system using the unique DCL4-dependent miRNA, miR839. By replacing the mature sequence in the miR839 precursor sequence with that of miR172, one of the most widely conserved miRNAs in angiosperms, we succeeded in expressing miR172 from a chimeric miR839 precursor in dcl1-7 plants and observed the repression of miR172 target gene expression. In parallel, the DCL4-dependent miR172 expression rescued the late flowering phenotype of $d c / 1-7$ by acceleration of flowering. We established the DCL1-independent miRNA expression system, and revealed that the reduction of miR172 expression is responsible for the $d c / 1-7$ late flowering phenotype.
\end{abstract}

Keywords: RNA; microRNA; Arabidopsis; development; DCL1; DCL4; miR839

\section{INTRODUCTION}

MicroRNAs (miRNAs) are endogenous noncoding RNAs 18-24 nt long, and are involved in RNA silencing by repression of the expression of target gene mRNAs having complementary sequences (Bartel 2004). In plants, a miRNA and ARGONAUTE 1 (AGO1), an effector protein of RNA silencing, constitute the major miRNA-induced silencing complex (miRISC) as the functional machinery (Iki et al. 2010). miRISCs bind to target mRNAs by miRNA guidance and suppress the target gene expression by cleavage or translational repression in plants (Llave et al. 2002; Brodersen et al. 2008; Li et al. 2013). The biogenesis of plant miRNAs occurs via two-step processing in nuclei: The first process converts the primary miRNA transcript (pri-miRNA) to the precursor miRNA (pre-miRNA), and the second process converts the pre-miRNA to mature 21-nt miRNA/miRNA* duplex. DICER-LIKE 1 (DCL1), a homolog of animal Dicer, is an RNase III-like protein and is the core enzyme of the two-step processing in collaboration with HYPONASTIC LEAVES 1 (HYL1) and SERRATE (SE) (Kurihara and Watanabe 2004; Kurihara et al. 2006; Yang et al. 2006).
In Arabidopsis thaliana, many miRNAs have important regulatory roles in various developmental events, including the vegetative-to-reproductive phase transition and the development of flowers and roots (Nag and Jack 2010; Huijser and Schmid 2011; Petricka et al. 2012). Although it is common that one animal miRNA targets more than 100 genes, plant miRNAs tend to target one or several particular mRNAs. Notably, many such target mRNAs encode transcription factors (Axtell 2008). These transcription factors, whose expression levels are balanced with cognate miRNAs, control cell differentiation and the cell division pattern during plant development.

Arabidopsis dcll mutants show a wide range of developmental defects (Schauer et al. 2002). Mutants of weak dcl1 alleles, like dcl1-7 (sin1-1: short integument1-1) and dcl1-9 (caf-1: carpel factory-1), show pleiotropic phenotypes including female sterility, late flowering, and small leaves. Null mutants, like dcl1-5 (sus1-5: suspensor1-5), are embryonic lethal. These developmental defects suggest that most if not all miRNAs are indispensable determinants for plant

(C) 2014 Tsuzuki et al. This article, published in RNA, is available under a Creative Commons License (Attribution-NonCommercial 4.0 International), as described at http://creativecommons.org/licenses/by-nc/4.0/. 
development. However, we do not know which species of miRNAs are required for plant development.

The expression levels of almost all miRNAs decrease in $\mathrm{dcll}$ mutants; contributions of individual miRNAs remain enigmatic. Double mutants between the $d c l 1$ mutant and a mutant of a target gene of the particular miRNA might provide a rare clue. Nodine and Bartel (2010) reported that dcl1-5, one of the $d c l 1$ null mutants, could be rescued by crossing with miR156-target SPL10-RNAi/spl11-1 mutant only with regard to the early embryonic patterning. However, this approach is restricted because the crossed mutant had mutations only in a part of the miRNA target genes, and has difficulty in crossing the mutants of other miRNAs also. Thus, to screen out the essential miRNA(s) for normal plant development directly, we need to recover $d c l 1$ mutants by decreased expression of miRNAs via an alternative pathway that is independent of DCL1.

In Arabidopsis, a few miRNAs are expressed independently of DCL1, unlike other general miRNAs. The expression of miR822, miR839, and miR859 are dependent on DCL4, one of the DCL1 homologs in plants (Rajagopalan et al. 2006; Ben Amor et al. 2009). Arabidopsis has four DCL proteins and they have distinct characters, respectively. Whereas DCL1 cognates a hairpin RNA structure of miRNA precursors, DCL2 and DCL4 produce endogenous and virus-derived small interfering RNAs (siRNAs) from long doublestranded RNAs (dsRNAs), which down-regulate the target RNA accumulation level. DCL3 also cognates dsRNAs and produces siRNAs working in RNA-directed DNA methylation. The exceptional substrate preference of DCL4 for the noncanonical DCL4-dependent miRNAs is considered to be due to their relatively long and high-complementary precursors (Chapman and Carrington 2007).

In this study, we established an artificial precursor construct based on a DCL4-dependent miRNA, miR839, to express a miRNA in $d c l 1$ mutants. To take advantage of the system, we tested miRNAs responsible for the $d c l 1$ mutant phenotypes in terms of phenotypic recovery. By replacing the mature sequence in the precursor of miR839 with the miR172 mature sequence, miR172 was expressed in a DCL4-dependent manner. miR172 processed with the aid of DCL4 could suppress the expression of the known miR172 target genes and rescue some, but not all, of the dcl1-7 late flowering phenotype. Thus, our study provided technical progress for future research, and the role of one miRNA in Arabidopsis development was clarified through miRNA complementation in the $d c l 1-7$ mutant.

\section{RESULTS}

\section{dcl1-7 and dc/1-9 mutants show distinct patterns in their miRNA expression profiles}

Some miRNA families are conserved widely among angiosperm species. It is accepted that DCL1 is involved in the pro- cessing of almost all miRNA precursors. Accordingly, many dcl1 mutants of different alleles show developmental phenotypes in flowering time and reproduction. It is suspected that such defects are caused by decreases in the levels of miRNAs with regulatory roles in plant development, and that the imbalance of target mRNAs accumulation causes uncoordinated cell differentiation and morphogenesis.

We suspected that weak allele mutants of $d c l 1$ have some biased inability to express some miRNAs, resulting in allelespecific phenotypic changes. Thus, we checked the expression levels of miRNAs of 12 conserved families (miR156, miR159/ 319, miR160, miR162, miR164, miR165/166, miR167, miR168, miR169, miR170/171, miR172, and miR390) in the inflorescences of mutants $d c l 1-7$ and $d c l 1-9$ (Fig. 1A) and in wild-type (WT) Col-0 plants. As a result of ANOVA, it was indicated that the levels of most miRNAs have a difference between WT, dcl1-7, and dcl1-9, except for miR168 $(P<0.05)$.

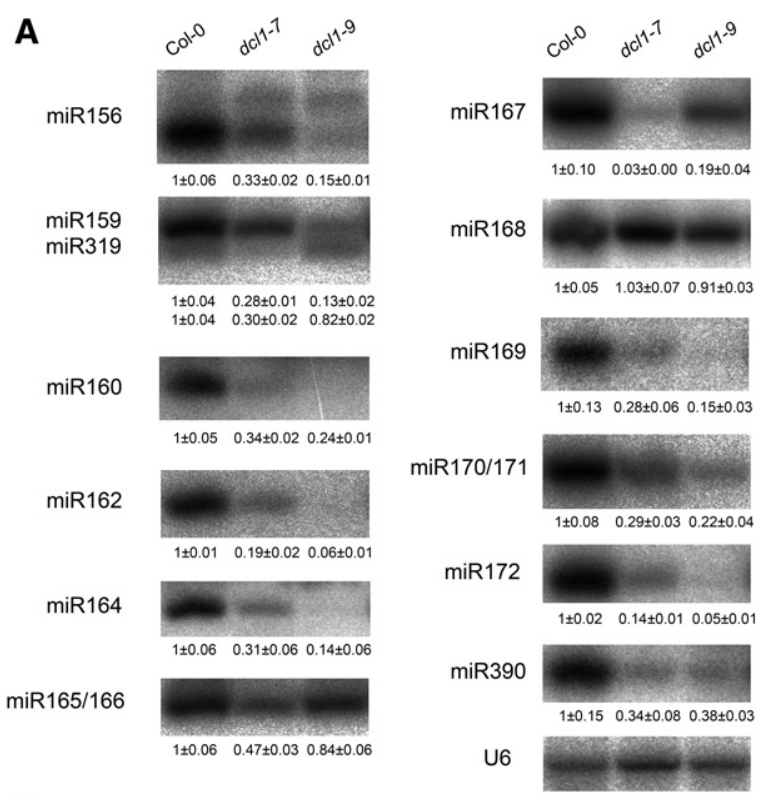

B

\begin{tabular}{|c|ccc|}
\hline & WT - dcl1-7 & WT - dcl1-9 & $d c / 1-7-d c 11-9$ \\
\hline miR156 & $*$ & $*$ & $*$ \\
\hline miR159 & $*$ & $*$ & $*$ \\
\hline miR160 & $*$ & $*$ & $(-)$ \\
\hline miR162 & $*$ & $*$ & $*$ \\
\hline miR164 & $*$ & $*$ & $(-)$ \\
\hline miR165/166 & $*$ & $(-)$ & $*$ \\
\hline miR167 & $*$ & $*$ & $(-)$ \\
\hline miR168 & $(-)$ & $(-)$ & $(-)$ \\
\hline miR170/171 & $*$ & $*$ & $(-)$ \\
\hline miR172 & $*$ & $*$ & $*$ \\
\hline miR319 & $*$ & $*$ & $*$ \\
\hline
\end{tabular}

FIGURE 1. Expression levels of miRNAs conserved widely among angiosperms were decreased in $d c l 1$ mutants. $(A)$ Northern blotting was used to detect the expressions of 12 miRNA families in $d c l 1-7$ and dcl1-9 inflorescences. The gel patterns are representatives of three experimental replicates, and the numbers below the gel pattern indicate relative fold levels averaged over three replicates and standard errors. U6 snRNA was used as the internal control. $(B)$ The table summarizes the results of statistic analysis of $A$ by Tukey's test $(n=3) .\left({ }^{*}\right) P<0.05$; $(-)$ no significant difference. 
Then, analysis using Tukey's test indicated that the levels of most miRNAs were reduced significantly in both $\mathrm{dcl} 1-7$ and $d c l 1-9$, except for miR165/166 and miR319 in dcl1-9 $(n=3, P<0.05)$ (Fig. $1 B)$. In addition, the two mutants showed distinct patterns of miRNA expression levels. The levels of some miRNAs (miR156, miR159, miR162, and miR172) were reduced more significantly in $\mathrm{dcl1}$ 9 than in dcl1-7. In contrast, the levels miR165/166 and miR319 were significantly higher in dcl1-9 than in dcl1-7 $(n=3, P<0.05)$ (Fig. 1B).

The MIR839A gene is possibly transcribed like other miRNA genes, and the transcript is processed independently of DCL1

Unlike other miRNAs, miR839 is expressed independently of DCL1, but is dependent on DCL4 (Rajagopalan et al. 2006). To confirm this, we performed Northern blotting. miR839 expression was detected at the same level in the three different mutants (dcl1-7, dcl1-9, and $d c l 1-100$ ), but not in $d c l 4-2$ (Fig. 2A), as reported in previous studies (Rajagopalan et al. 2006).

The MIR839A locus in the Arabidopsis genome is shown in Figure 2B. We performed RNA ligase-mediated rapid amplification of cDNA ends (RACE) to identify the transcription start site $(+1$ site), which was 735-nt upstream of the $5^{\prime}$-end of the mature miR839 sequence, and the termination site, which was 364nt downstream from the $3^{\prime}$ end of the mature sequence. It is possible that the primary transcript of the MIR $839 \mathrm{~A}$ gene is transcribed as other MIRNA genes.
A

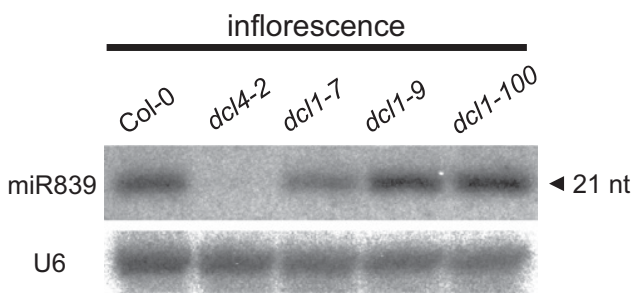

B

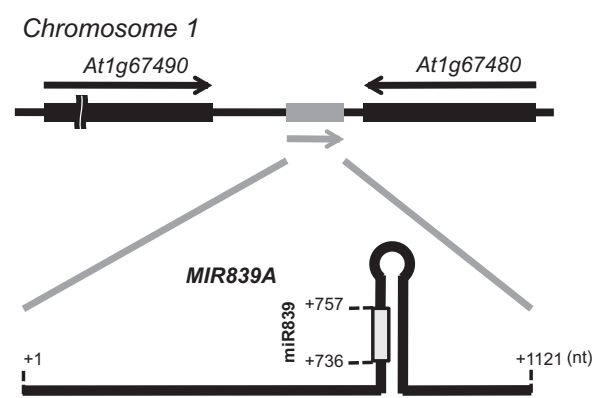

E

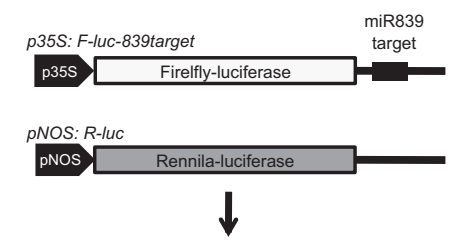

Agrobacterium-mediated transient expression in N. benthamiana and measure F-luc/R-luc level

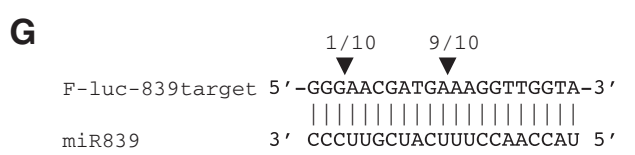

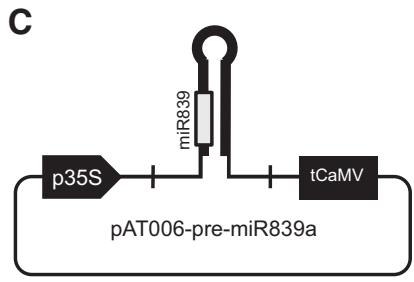

D

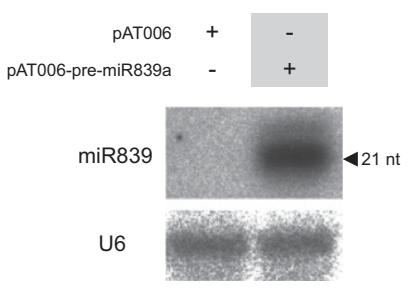

$\mathbf{F}$

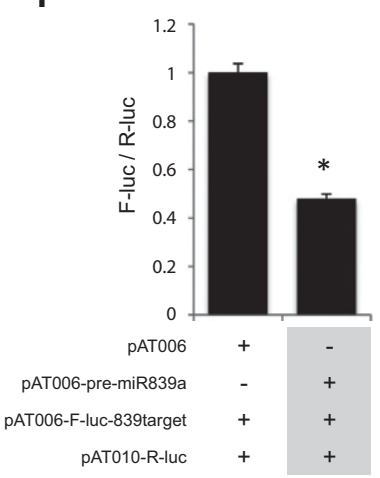

FIGURE 2. Characterization of DCL4-dependent miR839 expression revealed that miR839 has silencing ability. (A) Northern blotting to detect the expression of miR839 in florescences of $d c l 4$ and $d c l 1$ mutants. (B) Genome mapping of Arabidopsis MIR839A gene. Rapid amplification of cDNA ends (RACE) was used to determine the transcription start site and termination site, which are represented as +1 and +1121 , respectively. $(C)$ Illustration of the cloning of pre-miR839 into vector pAT006 and Agrobacterium-mediated transient expression in N. benthamiana. (D) Ectopic expression of miR839 in N. benthamiana mediated by Agrobacterium. (E) Illustration of the luciferase constructs used in Dual-luciferase reporter assay in N. benthamiana. $(F)$ Repression of firefly luciferase expression by miR839 ectopic expression in $N$. benthamiana. The mean fold value of F-luc/R-luc is represented $(n=4)$. Error bar, SE. $\left(^{*}\right) P<0.05$. $(G) 5^{\prime}$-RACE assay to determine the $5^{\prime}$ ends of the $3^{\prime}$ cleavage product of firefly luciferase mRNA. Arrowheads represent the $5^{\prime}$-end position and the number of RACE-clones.

\section{Ectopically expressed miR839 has gene-silencing ability like other miRNAs}

We then tested whether the miR839 precursor functioned like conventional miRNAs to suppress target mRNAs. For this purpose we chose a transient expression system using Nicotiana benthamiana leaves to observe whether simultaneous introduction of the miR839 gene could suppress ectopic expression of an mRNA with the miR839 target site at its $3^{\prime}$ UTR.

First, we cloned the genomic sequence of the miR839 precursor into plasmid pAT006 between the CaMV35S promot- er and terminator to obtain pAT006-pre-miR839a (Fig. 2C). pAT006-pre-miR839a was then transfected into N. benthamiana leaves. Two days later, we detected ectopic expression of miR839 in the N. benthamiana leaves (Fig. 2D). This result confirmed that the miR839 precursor was transcribed and processed correctly into the precise mature miR839.

We then confirmed whether or not miR839 was able to reduce the target gene expression. We checked the ability of miR839 to suppress its target mRNAs using the Dual-luciferase reporter assay in $N$. benthamiana leaves. The complete complementary nucleotide sequences of miR 839 were inserted downstream from the firefly luciferase gene (Fig. 2E). The 
result showed that the expression of firefly luciferase was significantly decreased in the presence of mature $\operatorname{miR} 839(n=$ $4, P<0.05$, Student's $t$-test), but not in its absence (Fig. 2F).

We also used 5' RACE to check whether or not the cleavage of firefly luciferase mRNA occurred precisely at the miR839 target site (Fig. 2G). The result showed that nine of 10 RACE-clones were cleaved at the AGO1 slicing position between nucleotides 10 and 11 of the miRNA sequence (Fig. 2G). Based on these results, we judged that the miR839 precursor could be used for ectopic expression, as reported using other miRNAs dependent on DCL1 (Parizotto et al. 2004).

\section{Precursor construct of miR839 could express a noncognate miRNA in a dcl1 mutant and suppress its target gene expression}

The miR839 precursor was not processed by DCL1, but possibly by DCL4, so we hypothesized that the miR839 precursor structure could be used to express otherwise DCL1-dependent miRNAs.

To test this, we designed a chimeric miR839 precursor sequence whose mature sequence and its complementary sequence (miRNA*) were easily replaced by specific miRNAs and their complementary sequences by introduction of BsaI restriction enzyme site (Fig. 3A,B). In addition, the $35 \mathrm{~S}$ promoter sequence was placed upstream of the artificial miR839 precursor sequence to drive its transcription. If this strategy worked, DCL1-dependent miRNAs could be expressed by DCL4 activity from the chimeric miR839 precursor in $d c l 1$ mutants.

miR172, which is one of the conserved miRNA families in angiosperms, has a role in flower development and the promotion of flowering time by repressing expression of the AP2 gene family (Aukerman and Sakai 2003; Chen 2004; Wu et al. 2009). Moreover, the level of miR172 was strongly decreased in the two dcl1 mutants that showed the late flowering phenotype (Fig. 1). Based on such observations and earlier results, we determined that miR172 was a candidate miRNA for the $d c l 1$ phenotype. We constructed a chimeric miR839 precursor construct in which the mature miR839 sequence was replaced with miR172a to obtain pWAT206-pre-miR839-172. To examine the efficiency of the pre-miR839-172 construct, we transformed $d c l 1-7$ heterozygous plants with this vector using Agrobacterium. After selection of T1 plants heterozygous for both the $d c l 1-7$ mutation and the 35S-pre-miR839-172 transgene, T2 seeds were harvested. Such seeds were planted and their flowering times observed. We selected two transgenic plant lines, designated \#1 and \#2. In general, the late flowering phenotype of $d c l 1-7$ was rescued in the 35S: pre-miR839-172/ dcl1-7 plants (Fig. 4A), while the sterile phenotype was not complemented (data not shown). Then we performed a statistical analysis comparing the flowering time of transgenic plants \#1 and \#2 with that of $d c l 1-7$. As a result, both transgenic lines \#1 and \#2 flowered significantly earlier than dcl1-7 plants $(P<0.001$, Mann-Whitney test with Bonferoni correction; F-test showed the inequality between variances) (Fig. 4B, C). This result, therefore, suggests that the transgene rescued the $d c l 1-7$ late flowering phenotype.

We then checked the expression of miR172 in the 35S-premiR839-172 transformants \#1 and \#2. Untransformed dcl1-7 
A

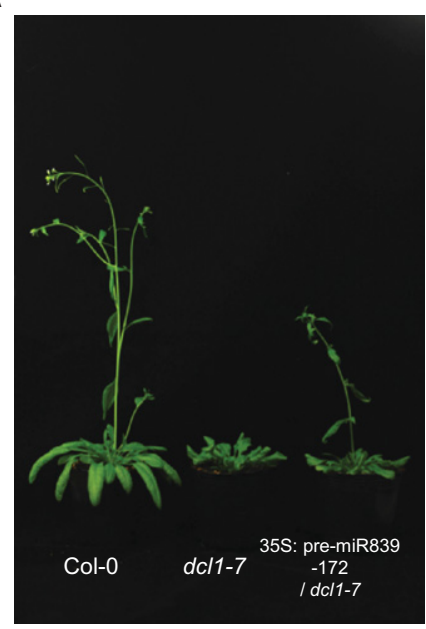

B

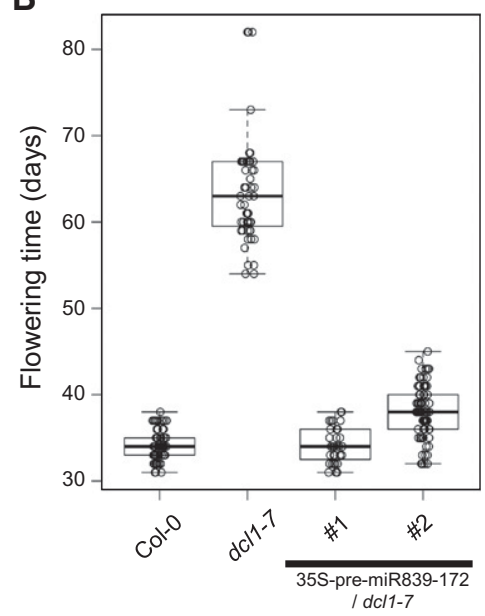

C

\begin{tabular}{|c|c|c|}
\hline Genotype & $\begin{array}{c}\text { Flowering time } \pm \mathrm{SE} \\
\text { (days) }\end{array}$ & $n$ \\
\hline Col-0 & $34.2 \pm 0.2$ & 72 \\
\hline$d c / 1-7$ & $63.2 \pm 0.8$ & 52 \\
\hline 35S-pre-miR839-172/dc11-7 \#1 & $34.1 \pm 0.3$ & 36 \\
\hline 35S-pre-miR839-172/dc/1-7 \#2 & $38.1 \pm 0.3$ & 80 \\
\hline
\end{tabular}

FIGURE 4. DCL1-independent expression of miR172 recovered the late flowering phenotype of dcl1-7. (A) Image of a dcl1-7 homozygous plant at $47 \mathrm{~d}$ showing rescued phenotype of late flowering by 35S-pre-miR839-172 transgene. White characters indicate each genotype. Wild-type (WT) and dcl1-7 plants without transgenes were used as controls. (B) Overlap dot plots with box plots showing the distribution of flowering time in each genotype. The top and bottom of the box show the first and third quartile each, and the band inside the box shows the second quartile. The ends of whiskers show the highest datum within the 1.5 interquartile range (IQR) of the upper quartile and the lowest datum within the 1.5 IQR of the lower quartile; \#1 and \#2 indicate the same lines as in Figure 3C. $(C)$ The table summarizes the flowering time shown in $B$. Numbers show the averages and standard errors.

accumulated a low level of miR172 and miR167. In contrast, 35S: pre-miR839-172/dcl1-7 \#1 and \#2 plants expressed a significantly higher level of miR172 than $d c l 1-7$, but not miR167 ( $n=3, P<0.01$, Tukey's test) (Fig. 3C). This result showed that the 35S: pre-miR839-172 transgene could express miR172, even in the absence of fully functional DCL1. The earlier flowering phenotype correlated with the expression of mature miR172.

We then checked the silencing ability of miR172 expressed in a DCL4-dependent manner. Both TOE1 and TOE2 are target genes of miR172, and have an effect on repression of flowering time (Aukerman and Sakai 2003). Using qRTPCR, we quantified the level of TOE1 and TOE2 mRNAs in 35S: pre-miR839-172/dcl1-7 \#1 and \#2 plants and compared them with those of WT and dcl1-7 plants. TOE2 mRNA in dcl1-7 significantly increased by $\sim 3.6$-fold compared with WT plants, whereas in 35S: pre-miR839-172/ dcl1-7 \#1 and \#2 plants, the TOE2 mRNA level was the same as that in the WT $(n=3, P<0.05$, Tukey's test) (Fig. 3D). In contrast, the level of TOE1 mRNA was not statistically different between WT, dcl1-7, and 35S-pre-miR839$172 /$ dcl1-7 plants ( $n=3, P>0.05$, ANOVA) (Supplemental Fig. S1).
Taken together, the results suggested that miR172 expressed from the premiR839-172 construct could suppress the target gene in the absence of full DCL1 function and resulted in an earlier flowering time. In addition, our data indicated that the decrease of miR172 expression level is the main cause of the late flowering phenotype of $d c l 1-7$; however, it is not the cause of other $d c l 1$ pleiotropic defects.

\section{DISCUSSION}

The study of specific miRNAs using alternative miRNA biogenesis pathways has been awaited in the analysis of miRNA biogenesis mutants (Yang and Lai 2011). In such cases, where the levels of most miRNAs decreased, it is difficult to analyze the contribution of respective miRNAs in detail. miRNA expression by a noncanonical pathway could overcome this problem and make it possible to test the roles of each miRNA. Until now, the ectopic expression of miRNAs by a noncanonical pathway has not been successful in plant cells, in contrast to animals (Maurin et al. 2012; Yang et al. 2012). In this study, we expressed miR172 in $d c l 1-7$ via the noncanonical DCL4-dependent biogenesis pathway using miR839 precursor backbone. This is the first report of the use of a noncanonical miRNA expression system in plants. Additionally, we also established transformants expressing one miRNA specifically via the noncanonical pathway and succeeded in detecting its direct role in vivo.

Knockout (KO) mutants of the main factors in the miRNA biogenesis pathway, such as dicerKO and droshaKO, often show severe developmental defect phenotypes in plants and animals (Schauer et al. 2002; Bernstein et al. 2003). In Arabidopsis, dcl1 null mutants are embryonic lethal, whereas weak dcl1 mutant alleles have a wide range of developmental defect levels. Although both dcl1-7 and dcl1-9, two such weak alleles, are sterile because female reproductive organs are developmentally defective, there is a slight difference between them; dcl1-7 shows abnormal ovule development, while dcl1-9 shows excessive cell proliferation in the third and fourth whorls of the floral organs (Schauer et al. 2002). Between these two alleles, we observed differences in the expression levels of widely conserved miRNA families (Fig. 1). These distinct expression patterns explained some, if not all, of the phenotypic differences between them.

As the first step for comprehensive understanding, we applied the system to express one miRNA in a $d c l 1$ mutant 
allele: miR172 in dcl1-7. miR172 is a well-known plant miRNA involved in flowering promotion and flower development (Aukerman and Sakai 2003; Chen 2004). The expression level of miR172 was low in $d c l 1-7$ (Fig. 1). In this study, miR172 was expressed in a DCL1-independent manner and could rescue the $d c l 1-7$ late flowering defect (Fig. 4A-C). Our data are consistent with miR172's role in the promotion of flowering. Thus, the DCL4-dependent miRNA biogenesis pathway could be used to complement canonical DCL1-dependent miRNAs. In addition, miR172 is functionally related to miR156, which has a reciprocal role in inhibiting maturation. SPL genes targeted by miR156 promote the expression of miR172 and this sequential relation induces maturation and flowering ( $\mathrm{Wu}$ et al. 2009). While both miR156 and miR172 decreased in dcl1-7 plants (Fig. 1), dcl1-7 shows the late flowering phenotype and the sole expression of miR172 rescued the late flowering phenotype (Fig. 4AC). This observation suggested that expression of miR172 is epistatic to the effect of miR156 in the determination of flowering time. Comparative genome analysis shows that miR156 is conserved among almost all land plants, whereas miR172 exists only in angiosperms, and not in mosses and lycopods (Axtell and Bartel 2005). Therefore, the regulatory system involving miR172 must have evolved during the environmental adaptation of angiosperms on land. In light of the physiological and morphological differences among them, it is thought that miR172 emerged in an ancestor of angiosperms, and then obtained a dominant role over miR156 in developmental timing for promotion of reproduction with seeds.

Finally, we obtained partial recovery of the $d c l 1-7$ phenotype by expressing miR172; however, we could not attain complete complementation of dcl1-7's sterile phenotype. To realize complete rescue of $d c l 1$ plant fertility, more miRNAs are required in addition to miR172. Taking advantage of our DCL1-independent miRNA expression system, stepwise rescue of these multiple $d c l 1$ mutant defects by specific miRNA will make it possible to dissect each miRNA's role in Arabidopsis developmental transition in detail. The identification of miRNAs indispensable for plant reproduction is important in both basic and applied plant research. Further studies are required to fulfill the promise of our developed DCL1-independent miRNA expression system.

\section{MATERIALS AND METHODS}

\section{Plant materials and growth conditions}

The dcl1-7 allele was kindly provided by James C. Carrington and is previously described (Xie et al. 2005). The dcl1-9 allele was backcrossed six times with Col-0 plants. dcl1-100 (GABI_098F10), dcl4-2 (GABI_160G05), and mir839a (SAIL_21_B06) seeds were obtained from the ABRC and NASC stock centers. WT plants were Columbia-0 (Col-0) ecotype.

Arabidopsis thaliana plants were grown on soil under $22^{\circ} \mathrm{C}$ longday conditions ( $16 \mathrm{~h}$ light/8 h dark) to analyze progression of devel- opment or continuous light for other analysis. All N. benthamiana plants were grown on soil at $24^{\circ} \mathrm{C}$ under continuous light.

\section{Plasmids construction}

Vectors pAT and pWAT were used for plant transformation in this study. Vector pAT has a pMDC background and has the same multiple cloning site as pGREEN II 0000. Vector pWAT is a modified version of pGREEN II 0000 and is previously described (Kumakura et al. 2013). Both pAT006 and pWAT206 have a CaMV 35S promoter and terminator in the multiple cloning site, while pAT010 has a NOS promoter and terminator. pWAT206 has a BASTA resistant gene.

The precursor sequence of miR839 was amplified from Col-0 genomic DNA and cloned into pAT006 via two restriction enzymes, SalI and SpeI, to obtain pAT006-pre-miR839.

The chimeric miR839 precursor was synthesized with the type IIS restriction enzyme BsaI (Fig. 3A). First, the PCR fragment containing the base of miR839 precursor sequence and two inverted BsaI sites was amplified by overlapping PCR and inserted into SalI-SpeI sites in pWAT206 to obtain pWAT206-pre-miR839Bsa. Second, the PCR fragment of chimeric pre-miR839-172 with the BsaI sites in both ends was amplified from pAT006-pre-miR839 and ligated into pWAT206-pre-miR839Bsa after BsaI cutting to obtain pWAT206-pre-miR839-172.

It was reported that miR156 acts on the target sequence of SPL3 mRNA at its $3^{\prime}$ UTR (Gandikota et al. 2007); it is a rare case in plant miRNA, because most miRNAs have target sequences in the coding regions of their target mRNAs. In consideration of the future analysis of this line, we tried to mimic the natural context for miRNA silencing to suppress the target sequence in the $3^{\prime}$ UTR of the mRNA. For this purpose, the $3^{\prime}$ UTR and following terminator sequence of SPL3 was cloned downstream from the stop codon of the firefly luciferase gene. The native miR156-target sequence was replaced with the miR839-complementary sequence, without any additional sequence insertion, using BsaI.

All primers used for construction are listed in Supplemental Table S1.

\section{Agrobacterium-mediated transient expression assay}

Agrobacterium tumefaciens (GV3101) was infiltrated into N. benthamiana leaves as described previously (Takeda et al. 2008). The concentration of Agrobacterium was normalized to $0.8 \mathrm{OD}_{600}$. Leaves were sampled $48 \mathrm{~h}$ after infiltration.

To estimate the silencing efficiency of miRNAs in plants, vectors containing firefly luciferase or Renilla luciferase gene as the internal control were coinfiltrated with the precursor of miRNA into N. benthamiana leaves by an Agrobacterium-mediated transient expression assay. The ratio of the suspension of Agrobacterium transformed by each vector (pAT006-pre-miR839 [or empty vector] : pAT006F-luc-839target : pAT010-R-luc) was 8:1:1.

\section{In planta transformation of Arabidopsis by Agrobacterium}

Agrobacterium in planta transformation was used to establish Arabidopsis transformants. Arabidopsis inflorescences were dipped in Agrobacterium transformed by a transgene-containing vector 
suspended in 5\% sucrose and 0.04\% Silwet L77 solution. $d c 11-7$ heterozygous plants were selected by genotyping for transformation.

\section{RNA extraction and small RNA Northern blot analysis}

The RNAiso Plus reagent (TaKaRa) was used to isolate total RNA from Arabidopsis inflorescence or N. benthamiana infiltrated leaves. A total of $10 \mu \mathrm{g}$ of total RNA was separated on a denaturing $17.5 \%$ polyacrylamide gel $(7 \mathrm{M}$ urea) in $0.5 \times \mathrm{TBE}$ buffer $(45 \mathrm{mM}$ Tris, 45 mM boric acid, 1 mM EDTA at pH 8.0). Separated RNA was electroblotted onto Hybond- $\mathrm{N}^{+}$membranes (GE Healthcare). Radiolabeled DNA oligonucleotide probes were constructed by end-labeling with $\left[\gamma^{-}{ }^{32} \mathrm{P}\right]$ ATP by using T4 polynucleotide kinase. Hybridization was performed at $42^{\circ} \mathrm{C}$ with PerfectHyb Plus (SIGMA Aldrich). U6 snRNA was used as the internal control. Primers used for the radiolabeled probe are listed in Supplemental Table S1.

\section{Dual-luciferase assay}

The activity of each luciferase was detected using the DualLuciferase reporter assay system and Glomax 20/20 Luminometer (Promega).

Forty-eight hours after infiltration, leaf discs were excised and placed in $1.5-\mathrm{mL}$ tubes as samples. The leaf discs were ground in liquid nitrogen, $300 \mu \mathrm{L}$ of $1 \times$ Passive Lysis Buffer was added and the samples were mixed thoroughly. Following centrifugation at $22,600 \mathrm{~g}$ at $4^{\circ} \mathrm{C}$ for $3 \mathrm{~min}, 4 \mu \mathrm{L}$ of supernatant was mixed into 40 $\mu \mathrm{L}$ of Luciferase Assay Reagent II and the firefly luciferase activity was measured. Forty microliters of Stop \& Glo Reagent was added and Renilla luciferase activity was measured. Passive Lysis Buffer, Luciferase Assay Reagent II, and Stop \& Glo Reagent are all parts of the Dual-Luciferase Reporter Assay System's kit. Four biological replicates were performed for each sample.

\section{RNA ligase-mediated RACE}

$5^{\prime}$ and $3^{\prime}$ RACE were performed using the GeneRacer Kit (Invitrogen; Life Technologies), according to the manufacturer's protocol with GeneRacer oligo dT primer. To determine the fulllength of the MIR839A transcript, total RNA from Col-0 inflorescences was used. To detect the cleavage sites in the miRNA target sequence of the firefly luciferase mRNA, treatment of total RNA from infiltrated $N$. benthamiana leaves was started from the RNA ligase reaction, except for the CIP and TAP reactions to select only cleaved RNA fragments. Primers used for PCR amplification are listed in Supplemental Table S1.

\section{Quantitative RT-PCR (qRT-PCR)}

qRT-PCR was used to quantify miRNA-target gene mRNAs. Total RNA was extracted from Arabidopsis inflorescences and the PrimeScript RT reagent Kit with gDNA Eraser (TaKaRa) was used to synthesize the cDNA according to the manufacturer's protocol. qPCR was performed with the StepOnePlus Real Time PCR System (Applied Biosystems; Life Technologies) using a KAPA SYBR Fast qPCR kit (KAPA BIOSYSTEMS) and reaction conditions as previously described (Motomura et al. 2012). ACTIN2 was used as the internal control gene. Expression levels were averaged by two technical replicates and three biological replicates. Error bars in the relevant figure represented the standard error of the mean. Primer sets for each PCR amplification are listed in Supplemental Table S1. Primers to detect TOE1 and TOE2 mRNA were previously described (Wu et al. 2009).

\section{SUPPLEMENTAL MATERIAL}

Supplemental material is available for this article.

\section{ACKNOWLEDGMENTS}

We thank Dr. James C. Carrington for providing the seeds of $d c l 1-7$ and the ABRC and NASC for providing the seeds of the mutant lines. This work was supported by Grants-in-Aid for Scientific Research on Priority Areas (Grant No. 23012008 to Y.W.), for Scientific Research on Innovative Areas (Grant No. 25120707 to Y.W.), for Young Scientists (B) (Grant No. 22780035 to A.T.) and for Challenging Exploratory Research (Grant No. 24658283 to A.T.) from the Ministry of Education, Culture, Sports, Science, and Technology, Japan. Additionally, this work was funded by a Sasagawa Scientific Research Grant (to M.T.) from The Japan Science Society.

Received February 21, 2014; accepted May 5, 2014.

\section{REFERENCES}

Aukerman MJ, Sakai H. 2003. Regulation of flowering time and floral organ identity by a microRNA and its APETALA2-like target genes. Plant Cell 13: 2730-2741.

Axtell MJ. 2008. Evolution of microRNAs and their targets: Are all microRNAs biologically relevant? Biochim Biophys Acta 1779: 725734.

Axtell MJ, Bartel DP. 2005. Antiquity of microRNAs and their targets in land plants. Plant Cell 17: 1658-1673.

Bartel DP. 2004. MicroRNAs: genomics, biogenesis, mechanism, and function. Cell 116: 281-297.

Ben Amor B, Wirth S, Merchan F, Laporte P, d'Aubenton-Carafa Y, Hirsch J, Maizel A, Mallory A, Lucas A, Deragon JM, et al. 2009. Novel long non-protein coding RNAs involved in Arabidopsis differentiation and stress responses. Genome Res 19: 57-69.

Bernstein E, Kim SY, Carmell MA, Murchison EP, Alcorn H, Li MZ, Mills AA, Elledge SJ, Anderson KV, Hannon GJ. 2003. Dicer is essential for mouse development. Nat Genet 35: 215-217.

Brodersen P, Sakvarelidze-Achard L, Bruun-Rasmussen M, Dunoyer P, Yamamoto YY, Sieburth L, Voinnet O. 2008. Widespread translational inhibition by plant miRNAs and siRNAs. Science 320: 11851190.

Chapman EJ, Carrington JC. 2007. Specialization and evolution of endogenous small RNA pathways. Nat Rev Genet 8: 884-896.

Chen X. 2004. A microRNA as a translational repressor of APETALA2 in Arabidopsis flower development. Science 303: 2022-2025.

Gandikota M, Birkenbihl RP, Höhmann S, Cardon GH, Saedler H, Huijser P. 2007. The miRNA156/157 recognition element in the $3^{\prime}$ UTR of the Arabidopsis SBP box gene SPL3 prevents early flowering by translational inhibition in seedlings. Plant J 49: 683-693.

Huijser P, Schmid M. 2011. The control of developmental phase transitions in plants. Development 138: 4117-4129.

Iki T, Yoshikawa M, Nishikiori M, Jaudal MC, MatsumotoYokoyama E, Mitsuhara I, Meshi T, Ishikawa M. 2010. In vitro assembly of plant RNA-induced silencing complexes facilitated by molecular chaperone HSP90. Mol Cell 39: 282-291. 
Kumakura N, Otsuki H, Tsuzuki M, Takeda A, Watanabe Y. 2013. Arabidopsis AtRRP44A is the functional homolog of Rrp44/Dis3, an exosome component, is essential for viability and is required for RNA processing and degradation. PLoS One 8: e79219.

Kurihara Y, Watanabe Y. 2004. Arabidopsis micro-RNA biogenesis through Dicer-like 1 protein functions. Proc Natl Acad Sci 101: 12753-12758.

Kurihara Y, Yuasa T, Watanabe Y. 2006. The interaction between DCL1 and HYL1 is important for efficient and precise processing of pri-miRNA in plant microRNA biogenesis. RNA 12: 206212.

Li S, Liu L, Zhuang X, Yu Y, Liu X, Cui X, Ji L, Pan Z, Cao X, Mo B, et al. 2013. MicroRNAs inhibit the translation of target mRNAs on the endoplasmic reticulum in Arabidopsis. Cell 153: 562-574.

Llave C, Xie Z, Kasschau KD, Carrington JC. 2002. Cleavage of Scarecrow-like mRNA targets directed by a class of Arabidopsis miRNA. Science 297: 2053-2056.

Maurin T, Cazalla D, Yang J-S, Bortolamiol-Becet D, Lai EC. 2012. RNase III-independent microRNA biogenesis in mammalian cells. RNA 18: 2166-2173.

Motomura K, Le QT, Kumakura N, Fukaya T, Takeda A, Watanabe Y. 2012. The role of decapping proteins in the miRNA accumulation in Arabidopsis thaliana. RNA Biol 9: 644-652.

Nag A, Jack T. 2010. Sculpting the flower; the role of microRNAs in flower development. Curr Top Dev Biol 91: 349-378.

Nodine MD, Bartel DP. 2010. MicroRNAs prevent precocious gene expression and enable pattern formation during plant embryogenesis. Genes Dev 24: 2678-2692.

Parizotto EA, Dunoyer P, Rahm N, Himber C, Voinnet O. 2004. In vivo investigation of the transcription, processing, endonucleolytic activ- ity, and functional relevance of the spatial distribution of a plant miRNA. Genes Dev 18: 2237-2242.

Petricka JJ, Winter CM, Benfey PN. 2012. Control of Arabidopsis root development. Annu Rev Plant Biol 63: 563-590.

Rajagopalan R, Vaucheret H, Trejo J, Bartel DP. 2006. A diverse and evolutionarily fluid set of microRNAs in Arabidopsis thaliana. Genes Dev 20: $3407-3425$

Schauer SE, Jacobsen SE, Meinke DW, Ray A. 2002. DICER-LIKE1: blind men and elephants in Arabidopsis development. Trends Plant Sci 7: 487-491.

Takeda A, Iwasaki S, Watanabe T, Utsumi M, Watanabe Y. 2008. The mechanism selecting the guide strand from small RNA duplexes is different among argonaute proteins. Plant Cell Physiol 49: 493500 .

Wu G, Park MY, Conway SR, Wang J, Weigel D, Poethig RS. 2009. The sequential action of miR156 and miR172 regulates developmental timing in Arabidopsis. Cell 138: 750-759.

Xie Z, Allen E, Wilken A, Carrington JC. 2005. DICER-LIKE 4 functions in trans-acting small interfering RNA biogenesis and vegetative phase change in Arabidopsis thaliana. Proc Natl Acad Sci 102: 1298412989.

Yang J-S, Lai EC. 2011. Alternative miRNA biogenesis pathways and the interpretation of core miRNA pathway mutants. Mol Cell 43: 892-903.

Yang L, Liu Z, Lu F, Dong A, Huang H. 2006. SERRATE is a novel nuclear regulator in primary microRNA processing in Arabidopsis. Plant J 47: 841-850.

Yang J, Maurin T, Lai EC. 2012. Functional parameters of Dicer-independent microRNA biogenesis functional parameters of Dicer-independent microRNA biogenesis. RNA 18: 945-957. 

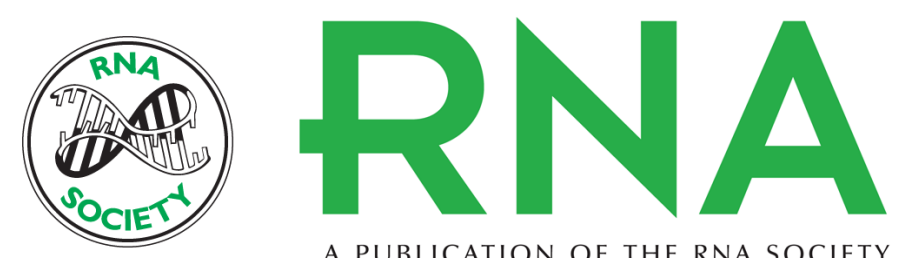

A PUBLICATION OF THE RNA SOCIETY

\section{Recovery of dicer-like 1-late flowering phenotype by miR172 expressed by the noncanonical DCL4-dependent biogenesis pathway}

Masayuki Tsuzuki, Atsushi Takeda and Yuichiro Watanabe

RNA 2014 20: 1320-1327 originally published online June 25, 2014

Access the most recent version at doi:10.1261/rna.044966.114

\section{Supplemental http://rnajournal.cshlp.org/content/suppl/2014/06/11/rna.044966.114.DC1 \\ Material}

References This article cites 31 articles, 14 of which can be accessed free at: http://rnajournal.cshlp.org/content/20/8/1320.full.html\#ref-list-1

Open Access Freely available online through the RNA Open Access option.

Creative This article, published in RNA, is available under a Creative Commons License Commons (Attribution-NonCommercial 4.0 International), as described at

License http://creativecommons.org/licenses/by-nc/4.0/.

Email Alerting Receive free email alerts when new articles cite this article - sign up in the box at the Service top right corner of the article or click here.

To subscribe to $R N A$ go to:

http://rnajournal.cshlp.org/subscriptions 